\title{
A rejoinder
}

\author{
DENNIS H. HOLDING \\ University of Louisville, Louisville, Kentucky 40206
}

As space restrictions preclude replying to many of the details of Dr. Coltheart's extensive restatement of the conventional iconic view, it is hoped that in most cases referring back to the original paper will be sufficient. This communication attempts to deal with some of the major issues where misunderstandings have arisen and with the proposed crucial experiment.

The cue delay argument does not support the visual image notion, unless its existence is already presupposed. It is as if one argued that it must be farther to the bank than to the post office, since it takes longer to get there. If it is indeed farther to the bank, it will probably take longer to get there but, if it is not, the travel time is irrelevant. As for the inference that both alternative explanations of partial report superiority "predict that rapid decay will not occur," it is difficult to see how this is derived. The cue anticipation explanation, at least, is completely neutral to the rapid decay issue, predicting neither one way nor the other. It seems likely that the decay effects are due to quite different factors, perhaps including rehearsal uncertainty, but more analytic research is needed to settle the issue.

The finding of near equality between partial and whole estimates of letter/number displays is ambiguous, yielding points for both sides. For the iconic view, it suggests that the output interference effect is too small; for the iconoclastic view, it suggests that the nature of the stimulus array precludes cue anticipation. The only recourse is to other evidence. However, the statement in the summary that other results "indicate that output interference effects are absent or negligible in tachistoscopic experiments" cannot be accepted, since the effects have been carefully analyzed and exhibited (Dick, 1971).

The direct investigations of visual persistence have revealed no evidence for excess capacity. It is true that Haber and Standing (1970) used a 3 by 3 matrix, but no information was extracted from the display. The notion that my paper "contends that, even if there is a brief postexposural visual memory, Ss cannot process this memory selectively" is quite misconceived. Ss may select as they wish from the few letters available to them. My contention, based on the evidence presently available, is that the other items from the stimulus array are simply not there. As for the form taken by the trace, it may be that it is central but unprocessed and visual and that it yields information for $2 \mathrm{sec}$ with a dark postexposure field but is only liable to summation for $100 \mathrm{msec}$, but adding that it is sensitive to interruption by its duplicate makes for a very complex proposition.

The idea of a crucial experiment is appealing. Unfortunately, the proposed experiment misses the mark, since both sides predict virtually the same result. Assuming that the cue sequences are taken straight from random number tables, separately for each $S$ and preferably with partial and whole cues randomized together, cue anticipation will play no part. No difference would be expected on these grounds, whether the visual noise mask was present or not. Of course, if any cuing bias is introduced, it should affect both masked and nonmasked conditions. Output interference should make for a small difference but, unless $\mathrm{N}$ is very large, the difference will be swamped by variability. Choosing a 3 by 3 matrix will reduce the sensitivity of the experiment, because Ss will be uncertain and variable in deciding how to allocate their extra letter. Adding the noise mask will make for further variability, since Ss vary enormously in their ability to disregard a backward mask.

The most important snag is that the visual noise will tend to reduce the overall scores. This will not be an erasure effect but, at least for unpracticed Ss, there will be some kind of interference. As Darwin (1971) says of a nonspecific form of auditory backward masking, "a kick on the shins may be an effective backward mask to this extent [p. 389]." Backward masking yields rather complex data for interpretation, but it does not matter to the present argument how its effect is achieved. It probably does matter how much practice has been given, at least if a visual backward mask behaves like its auditory counterpart. Loeb and Holding (1974) have recently found that the auditory backward masking effect, on which the preperceptual image hypothesis depends, is completely abolished by extended practice over several thousand trials.

However, let us assume that the conditions of the proposed experiment will be such as to produce poorer performance. Since the amount of output interference depends upon the amount of output, the interference will be reduced. We can estimate the amount of the reduction from Dick's (1971) data. The data show the output interference curve to be nonlinear, such that the decrement over the first two items is much less than the decrement over the third through sixth items. Thus, if the noise mask reduces the whole report scores to about two items, the putatively crucial experiment will be 
reduced to searching for a discrepancy amounting to some fraction of one letter.

\section{REFERENCES}

Darwin, C. J. Dichotic backward masking of complex sounds. Quarterly Journal of Experimental Psychology, 1971, 23, 386-392.

Dick, A. O. On the problem of selection in short-term visual (iconic) memory. Canadian Journal of Psychology, 1971, 25, 250-263.

Haber, R. N., \& Standing, L. G. Direct estimates of the apparent duration of a flash. Canadian Journal of Psychology, 1970 , 24, 216-229.

Loeb, M., Holding, D. H. Effects of practice on the degree of backward interference by tones or noise on pitch perception. Paper presented at the Southern Society for Philosophy and Psychology, 66 th Annual Meeting, Tampa, 1974. 